\title{
Prevalence of Human Immune Deficiency Virus in Suspected Tuberculosis Patients Attending Clinics in Benin City NIGERIA
}

\author{
L.E. Okoror*, F.I. Esumeh, P.I. Umolu, A. Enaigbe, R. Akpe, H.A. Obiazi, F. Aisabokhale and \\ I. Imahia
}

Department of Microbiology, Faculty of Natural Sciences, Ambrose Alli University, PMB 14, Ekpoma. Nigeria

\begin{abstract}
Tuberculosis and HIV have been closely linked since the emergence of acquired immune deficiency syndrome. HIV has contributed to a significant increase in the incidence of tuberculosis worldwide by producing a progressive decline in cell immunity. Four hundred and seventy sputum samples were collected from different clinics in and around Benin City and tested for tuberculosis and HIV. Of the 470 samples tested, only 147 (32\%) were positive while 323 (68\%) were negative for tuberculosis. $71(15 \%)$ were positive for HIV alone while $56(11 \%)$ were co-infected with both tuberculosis and HIV. Of the 103 patients who received BCG at childhood 18.2\% tested positive. More males tested positive than their female counterparts, while traders had the highest number of positive case and also had the highest attributable risk. Oredo Local Government Area of Edo State Nigeria had the highest number of positive cases. It could be said that HIV has contributed to the prevalence of tuberculosis in Edo State Nigeria. The high rate of tuberculosis is then associated with increase in HIV infection.
\end{abstract}

\section{INTRODUCTION}

Tuberculosis and HIV - have been closely linked since the emergence of the acquired immune deficiency syndrome, HIV infection had contributed to a significant increase in the worldwide incidence of tuberculosis by producing a progressive decline in cell immunity. HIV alters the pathogenesis of tuberculosis, greatly increasing the risk of developing diseases in co-infected individuals and leading to more frequent extra pulmonary involvement and atypical radiographic manifestation [1]. Although HIV- related tuberculosis is both treatable and preventable but incidence rates continue to climb in developing nations where HIV infection and tuberculosis are endemic and resources are limited. Worldwide, tuberculosis is the most common opportunistic infection affecting HIV - seropositive individuals and it is the most common cause of death in patients with AIDS [1].

Approximately 10 million people are estimated to be coinfected with M. tuberculosis and HIV, and over $90 \%$ of those dually infected individuals are in developing countries. In some areas of sub-Saharan Africa, the rates of co infection exceed 1,000 per 100,000 people [2,6]. Tuberculosis is the most common cause of death among patients with AIDS, killing 1 of every 3 patients worldwide [2]. After decades of steady decline, tuberculosis cases increased in 1986 in the United States, between 1985 and 1990, tuberculosis cases increased by $20 \%$, resulting in 28040 excess cases of tuberculosis. CDC [3] reported that AIDS- related tuberculosis accounted for a minimum of $30 \%$ of these excess cases. The decline in HIV-related tuberculosis in the US and other industrialized countries has parallel an overall decline in tuber-

\footnotetext{
*Address correspondence to this author at the Department of Microbiology, Faculty of Natural Sciences, Ambrose Alli University, PMB 14, Ekpoma, Nigeria; E-mail: LEOkoror@gmail.com
}

culosis cases. Whether or not the use of effective antiretroviral therapy (ART) has hastened this decline is not clear [6]. Two cohort studies have described the frequency of tuberculosis during the current treatment of HIV [4]. However the rate of tuberculosis, although low remains stable over the period studied. In contrast, the Euro SIDA cohort study of $7,000 \mathrm{HIV}$ infected patients reported dramatic declines in the rate of tuberculosis and disseminated Mycobacterium avium complex (MAC) from the period before 1993 to the period after 1997, coinciding with the introduction of potent ART. Further more, a separate study of 5,000 suspected tuberculosis patient showed that the number of males positive to tuberculosis was about twice the number of females. For example one hundred and fifty of the patients were positive to tuberculosis out of which 98 of them were males and $52 \mathrm{fe}$ males. However, no scientific reason was advanced for this discrepancy in tuberculosis infection [5].

Tuberculosis can develop through progression of recently acquired infection (primary disease), reactivation of latent infection, or exogenous re-infection. Recent data suggest that in urban areas within the United States, recent transmission accents for a larger proportion of cases than was realized previously [7]. Molecular genotyping in San Francisco and New York reported that $30 \%-40 \%$ of new cases were due to recent infection with rapid progression to disease. In both studies HIV infection or AIDS was an independent risk factor for recent acquisition of infection and rapid progression to disease [7].

Infection with $M$. tuberculosis can occur when an individual who is exposed to an infectious case of tuberculosis inhales particles $(<5 \mu \mathrm{m}$ in size) containing the tubercle bacilli. If the bacilli reach the pulmonary alveoli, they may be ingested by alveolar macrophages, which is the first line of defense against $M$. tuberculosis. Surviving tubercle baclli 
multiplies within the macrophages and eventually undergoes hematogenous spread to other areas of the body. Although defects in macrophages function have been demonstrated in HIV-infected patients, there is no conclusive evidence that HIV-seropositive person are more likely to acquire tuberculosis infection than HIV-seronegative individuals, given the same degree of exposure [8]. Once infection dose occur, however, the risk of rapid progression is much greater among persons with HIV infection, because HIV impairs host's ability to contain new tuberculosis infection. Immune competent individuals with $M$. tuberculosis have approximately a $10 \%$ life time risk of developing tuberculosis with half of the risk occurring in the first 1-2 years after infection; in contrast a San Francisco study reported rapid spread of tuberculosis during an outbreak in residential care facility for HIV- seropositive substance abusers [9]. In Nigeria, where most of the population lives on about $\$ 1.00$ a day, the cost of ART may be beyond the reach of most people infected with HIV. The delay in the introduction of ART may have also influenced the control of HIV and thereby tuberculosis.

Several cohort studies have demonstrated a high incidence of active tuberculosis among HIV infected individuals; however, the rate of active tuberculosis varies from population and region [10] hence this study therefore, screened individuals suspected to have tuberculosis for HIV to actually ascertain the prevalence. Only very little has been reported on HIV and tuberculosis co-infection from the study population. It is also aimed at knowing the involvement of $\mathrm{HIV}$ in the development of tuberculosis in the population.

\section{MATERIALS AND METHODS}

\section{Sample Collection}

Four hundred and seventy sputum samples were collected from patients with suspected cases of tuberculosis in Benin City. Patients on antiretroviral drugs were also included in the study. The sputum were collected in lick-proof sterile containers with transport medium and taken to laboratory for immediate analysis. Information such as age, sex, occupation, household size, previous vaccination against TB, previous TB treatment, drinking and smoking habit, area of residence were collected from the patients.

\section{Procedure}

The sputum were fixed on clean grease free slides and stained with the Ziehl-Neelsen staining technique [11]. The slides were then observed under the oil immersion objective of binocular light microscope. The samples were then processed by transferring 1-2 $\mathrm{ml}$ of sputum into a sterile universal screwed cap bottles. Equal volume of $5 \% \mathrm{NaCl}$ was added to the sputum, homogenized and left at room temperature for 10-15 minutes and vortexed at intervals to break the mucus in the sputum. About $8 \mathrm{ml}$ of sterile distilled water was added and centrifuged at 3,000 rpm for 15 minutes. The supernatants were discarded and the sediments were used to inoculate culture slopes. The Lowestein- Jensen medium used in making culture slopes was prepared according to the method of Marks and Zaher [11]. All sediments were inoculated onto Lowestein -Jensen slants and incubated (Gallenkamp) for 6 weeks at $37^{\circ} \mathrm{C}$ (Cheesbrough, 2002). All the patients were again screened for Human Immuno Deficiency
Virus (HIV). Venal blood samples were collected from the patients and centrifuged (hetituch) at 3,000 rpm Sera were separated and HIV screening was the carried out using the rapid spot test kit (Global) as directed by the manufacturer. A confirmation of positive cases was carried out using the ELISA technique as described by [12]. Statistical analysis was carried out using student t test of SPSS version 11 and chi square analysis. Approximation was done for odd ratio.

\section{RESULTS}

Of the 470 patients screened for $M$. tuberculosis, only $32 \%$ were positive. Traders had the highest positive cases $(25 \%)$ while teachers and full time house wives screened were negative (Table 1).

Regional distribution shows that most of the positive cases were from Oredo LGA of Edo state (53.1\%) while Ehor, Esan and Owo (Ondo State) gave no positive results (Table 4). Table 2 shows that more males have been infected with tuberculosis than females with age group 21-40 having the highest positive cases. The distribution of HIV among tuberculosis positive patients is shown in Table $\mathbf{4}$ where more males were infected with both HIV and tuberculosis and age group 21-40 years of age had the highest positive cases for both males and females. The general statistics for co-infection was that $52(11 \%)$ of the 470 patients screened, while $71(15 \%)$ had HIV alone.

Of the 22 patients who received BCG vaccine at childhood only $18.2 \%$ tested positive for tuberculosis. The incidence rate was highest for bricklayers and electricians who were followed by wood workers and traders. Relative risk for hair dressers was (4.1) and traders (3.2) and were the were highest, however, attributable risk for traders was highest (18.2\%) (Table 5). All the 19 specimens that tested positive for Ziehl-Neelsen stain were also positive for culture except 2 which did not give significant growth.

\section{DISCUSSION}

The high incidence of HIV among tuberculosis patients infers that the emergence of the HIV epidemic in the study population would have influenced the high prevalence of tuberculosis. A total of 150 patients $(32 \%)$ testing positive for tuberculosis also implies that the increase in the infection is a source of public health concern.

The immune system of individuals would have been brought down due to the emergence of HIV hence paving way for tuberculosis epidemics. Of the total positive results, the prevalence of the infection in males is higher and this may not be unconnected with the fact that the sampling method used gave more bias to males than females. This was so because of the occupational sampling gave males more number of cases against the females since most of the occupation sampled were more males oriented. However, statistical analysis using the student $t$ test of SPSS version 11 at 99\% CI shows that there was no significant difference between the numbers of males sampled with the number of females.

Age group 21-30 years had the highest number of positive cases to tuberculosis and this age group coincides with the very active age group both occupationally and sexually. 
Table 1. Occupational Distribution of Pulmonary Tuberculosis in Edo State Nigeria

\begin{tabular}{|c|c|c|c|c|}
\hline Occupation & Cases & Control & Cases & Control \\
\hline Driving & 38 & 75 & $24(15.6 \%)$ & 28 \\
\hline Teaching & 28 & 56 & 0 & 0 \\
\hline Student & 61 & 122 & $14(9.4 \%)$ & 24 \\
\hline Wood workers & 33 & 66 & $14(9.4 \%)$ & 24 \\
\hline Banking & 24 & 48 & $5(3.1 \%)$ & 5 \\
\hline Hair Dressing & 28 & 56 & $9(6.3 \%)$ & 5 \\
\hline Trading & 99 & 197 & $38(2.5 \%)$ & 24 \\
\hline Tailoring & 24 & 47 & $5(3.1 \%)$ & 5 \\
\hline House wife & 28 & 56 & 0 & 0 \\
\hline Bricklaying & 9 & 19 & $9(6.3 \%)$ & 5 \\
\hline Electricians & 5 & 9 & $5(3.1 \%)$ & 0 \\
\hline Total & 470 & 940 & 147 & 158 \\
\hline
\end{tabular}

Table 2. Age Group Distribution Of Pulmonary Tuberculosis in Edo State Nigeria

\begin{tabular}{|c|c|c|c|c|}
\hline \multirow{2}{*}{ Age } & \multicolumn{2}{|c|}{ Female } & \multicolumn{2}{c|}{ Male } \\
\cline { 2 - 5 } & No. Tested & No. Positive & No. Tested & No. Positive \\
\hline \hline $0-20$ & 28 & $5(3.1 \%)$ & 38 & $24(6.3 \%)$ \\
\hline $21-30$ & 75 & $24(1.6 \%)$ & 47 & 71 \\
\hline $31-40$ & 56 & $9(6.3 \%)$ & 94 & $47(12.5 \%)$ \\
\hline $41-$ & 61 & $14(9.4 \%)$ & $\mathbf{2 4 9}$ & $\mathbf{9 9}$ \\
\hline
\end{tabular}

Table 3. Age Group Distribution of HIV Among Tuberculosis Patients

\begin{tabular}{|c|c|c|c|c|}
\hline \multirow{2}{*}{ Age } & \multicolumn{2}{|c|}{ Female } & \multicolumn{2}{c|}{ Male } \\
\cline { 2 - 5 } & $\begin{array}{c}\text { No. Tested } \\
\text { With TB }\end{array}$ & $\begin{array}{c}\text { No. Positive } \\
\text { For HIV }\end{array}$ & $\begin{array}{c}\text { No. Tested } \\
\text { With TB } \\
\text { For HIV }\end{array}$ \\
\hline \hline $0-20$ & 5 & 5 & 9 & 24 \\
\hline $21-30$ & 24 & 14 & 19 & 0 \\
\hline $31-40$ & 9 & 5 & 47 & 0 \\
\hline $41-$ & 14 & 0 & $\mathbf{2 4}$ & $\mathbf{9 9}$ \\
\hline Total & $\mathbf{5 2}$ & $\mathbf{2 4}$ & 0 \\
\hline
\end{tabular}


Table 4. Geographical Distribution of Pulmonary Tuberculosis in Edo State Nigeria (By Local Government Area (L.G.A))

\begin{tabular}{|c|c|c|}
\hline L.G.A. & No. Tested & No. Positive \\
\hline \hline Oredo & 226 & $80(53.1 \%)$ \\
\hline Egor & 103 & $33(21.9 \%)$ \\
\hline Ikpoba-Okha & 61 & $14(9.4 \%)$ \\
\hline Ehor & 14 & 0 \\
\hline Etsako & 14 & 0 \\
\hline Uhumwode & 9 & $5(3.1 \%)$ \\
\hline Esan & 5 & $5(3.1 \%)$ \\
\hline Owan & 5 & $5(3.1 \%)$ \\
\hline Outside the State: & & $5(3.1 \%)$ \\
\hline Warri & 19 & $5(3.1 \%)$ \\
\hline Sapele & 5 & 0 \\
\hline Ika & 5 & \\
\hline Owo & 1 & \\
\hline
\end{tabular}

Since they are more involved in human interactions chances are that they become more vulnerable to infections than any other age groups. They also form a very active group sexually and may have contacted tuberculosis or become more vulnerable to tuberculosis due to the presence of HIV which they may have contacted during indiscriminate sexual behav- ior. This could be so with the high number of HIV cases in this group and also co-infection with tuberculosis.

Occupational distribution reveals that more traders tested positive to tuberculosis than any other occupation. This could however be because the traders are very close to the public and trading in Nigeria is usually done in unhealthy, often overcrowded environment which has earlier been reported as one of the major risk to contacting tuberculosis. This is because overcrowding allows for easy pick-up of the droplets of tuberculosis due decreasing air flow. During trading in this environment droplets of tuberculosis could be released into the air and later picked up by healthy individuals since they are all very close to each other in the market place, the dusty environments of the markets also enhances the transmission of the tubercle droplets by infected individuals and healthy individuals could pick up the droplets and get infected. Traders often travel out of their area of domicile for trading purposes and more frequently and these areas could be are of high endemicity and thereby increases their risk of contacting the infection and this could however account for the high relative risk observed for traders.

A high attributable risk of $18.2 \%$ (Table 5) implied that traders are highly exposed to the infection. Whether HIV infection contributed to the high risk in this group of occupation is still not known. Drivers, Civil Servants, Tailoring, Hair Dressing and Banking had the second highest relative risk, this does not however, mean that they are more exposed than others since their attributable risks was lower than some of the occupations. They however, have some risk of exposure. Hair dressers are normally involved with sharp objects and could have needle stick injuries and thereby are exposed to having HIV/AIDS which could also be a vehicle for transmission of tuberculosis being immunocompromised due to HIV infection.

Table 5. Occupational Risk of Pulmonary Tuberculosis in Edo State Nigeria

\begin{tabular}{|c|c|c|}
\hline Driving & $1.7 / 0.63$ & 11 \\
\hline Farming & $1.3 / 0.4$ & 6.2 \\
\hline Students & $1.2 / 0.23$ & 5.0 \\
\hline Banking & $2.0 / 0.2$ & 9.0 \\
\hline Hair Dressing & $4.1 / 0.33$ & 22 \\
\hline Catering & $2.0 / 0.2$ & 9.0 \\
\hline House wives & $0 / 0$ & 0 \\
\hline Bricklaying & $0.4 / 1.0$ & $\propto$ \\
\hline Electricians $(\chi=2.360, \mathrm{CI}=99 \%)$ & $\propto / 1.0$ & $\propto$ \\
\hline
\end{tabular}


Another high risk group is the students which are highly sexually active and stand the risk of contacting HIV and subsequently tuberculosis. It has been earlier reported that HIV increases the risk of tuberculosis and that it is easier to contact any infection on epidemic scale for a HIV positive individual [3]. It could generally be noted that occupation plays a very important role in transmission of tuberculosis as well as HIV.

It was also observed that infections throughout the regions sampled were not evenly distributed (Table 4) and were statistically different using the student $t$ test of SPSS version $11(\mathrm{CI}=99 \%)$. This difference varies from Local Government to Local Government Areas. And this could be as a result of different prevalent rate due to difference in prominent occupation and other social activities. Urbanization could also be a main reason as to why there is high prevalence; urbanization brings about increase in sexual activities, overcrowding and commercial sex. Co-occurrence of tuberculosis with HIV was high $(34.4 \%)$, this goes to show that HIV has adversely contributed to the high prevalence of tuberculosis in the population as earlier reported [9, 13]. This is possible because HIV causes the patients to be immunocompromised thereby causing the patient to loose protection against the invading pathogen. It has also been reported that tuberculosis infection can aid the HIV because purified protein derivative (PPD) can also increase viral replication in infected T lymphocytes and monocytes [14]. They also demonstrated a 5 to 160 fold increase in viral replication during the acute phase of untreated tuberculosis. The significance of this increase in viral load is uncertain. There were also a significant percentage of those who received BCG and still came up with tuberculosis. Immunity would have waded off and chances are that HIV would have affected the patients' immunity thereby allowing tuberculosis to proliferate, thereby rendering the vaccination useless. It could also be that the vaccine did not offer protection due to improper handling of the vaccine, there could be vaccine failure due the use of non-existing strain of the organism used in vaccine preparation in endemic areas. However, whether tuberculosis contributed to high prevalence of HIV in the population still remains a subject for further research.

\section{REFERENCES}

[1] Raviglione MC, Narain JP, Kochi A. HVI-associated tuberculosis in developing countries: Clinical features diagnosis and treatment. Bull WHO 1995; 70: 515-21.

[2] Dye C, Scheele S, Dolin P, et al. Global burden of tuberculosis. Estimated incidence, prevalence and mortality by Country. JAMA 1999; 282: 677-86.

[3] Centres for Disease Control and Prevention. Updated guidelines for the use of rifabutin for the treatment $o$ and prevention of tuberculosis among HIV-infected patients taking protease inhibitors. MMWR 2000; 49: 185-200.

[4] Ole K, Gatell JM, Mocroft A. Infections with Mycobacterium tuberculosis and Mycobacterium avium among HIV infected persons after introduction of highly active retroviral therapy. Am J Respir And Crit Care Med 2000; 162: 867-72.

[5] Worge MP, Catell JM, Ole K. Distribution pattern of tuberculosis. J Infec Dis 1998; 342: 43-50.

[6] Brost HR, Kamps BS, Gute P. Changing pattern of AIDS-defining illness in the era of anti-retroviral combination therapy. AIDS 1999; 11: 1731-8.

[7] Small PM, Hopewell PC, Singh SP, et al. The epidemiology of tuberculosis in San Francisco. A population base study using conventional and molecular method. N Engl J Med 1994; 330: 1703-9.

[8] Meltzer MS, Skillman DR, Gomatos PJ, et al. Role of mononuclear phagocytes in the pathogenesis of HIV infection. Ann Rev Immunol 1990; 8: 169-94.

[9] Daley CL, Small PM, Schecter GF. An outbreak of tuberculosis with accelerated progression among persons infected with HIV: an analysis using restriction fragment length polymorphism. N Engl Med J 1992; 326: 321-35.

[10] Antionucci G, Girardi E, Raviglione MC. Risk factor of tuberculosis in HIV-infected persons. A prospective cohort study. JAMA 1995; 274: 143-8.

[11] Cheesbrough M. District Laboratory Practice in Tropical Countries Part 2. Cambridge University Press. UK. Last Page.

[12] Krivoshein YS. Handbook on Micrbiology Laboratory Diagnosis of Infectious Diseases. MIR Publishers, 1989; Moscow. 319 pp.

[13] American Thoracic/centre for Disease Control and Prevention. Infectious Diseases Society of America. Treatment of tuberculosis. Am J Respir Crit Care Med 2003; 4: 630-7.

[14] Goletti D, Weissman D, Jackson RW. Effect of Mycobacterium tuberculosis on HIV replication. J Immunol 1996; 157: 1271-8. 